\title{
Jean Itard en clave de educación inclusiva
}

\author{
Manuel Lopez-Torrijo* \\ Fran J Garcia-Garcia** \\ Jesús López García**
}

\section{Resumen}

Jean Itard ha sido valorado tradicionalmente como el padre de la Educación Especial. Un análisis de sus Informes de 1801 y 1807 sobre la educación de Víctor, el joven salvaje de L'Aveyron desde la perspectiva de la Educación Inclusiva permite descubrir en él algunos rasgos significativos que ayudan a considerarlo como un claro precursor de ésta. Tales son: su reivindicación del derecho a la educación de los más desfavorecidos y la aplicación de un programa de formación integral, así como de un plan de rehabilitación individualizado a partir de las capacidades, limitaciones, necesidades y aspiraciones del alumno. Sus aportaciones metodológicas adelantan algunas de las propuestas actuales de la educación inclusiva: importancia de la atención temprana, valor del trabajo interdisciplinar, la integración social del individuo como última meta de la educación. Pese al fracaso en su intento de ofrecer el habla a Víctor, las propuestas y reivindicaciones de Itard acerca del derecho de un joven salvaje a una educación atendida por administradores y científicos, y auspiciada por el propio gobierno son un claro precedente de algunas propuestas básicas de la Educación Inclusiva que orienta en la actualidad las principales Declaraciones Internacionales.

Palabras claves: Itard; Educación inclusiva; Educación especial.

* Professor doutor da Universidade de Valência, Valência, Valência, Espanha.

** Graduado em Educação social pela Universidade de Valência, Valência, Valência, Espanha.

*** Licenciado em Ciências da atividade física e do esporte pela Universidade de Valência, Valência, Valência, Espanha. 


\section{Jean Itard from the inclusive education viewpoint}

\section{Abstract}

Jean Itard has traditionally been valued as the father of Special Education. An analysis of his reports in 1801 and 1807 about the education of Victor, the wild child of L'Aveyron, from the perspective of inclusive education allows discovering in him some significant features that permit to consider him as a clear precursor of it. These are: his claims to the right to education of the disadvantaged, the implementation of a comprehensive training program and an individualized rehabilitation plan beginning with the abilities, limitations, needs and aspirations of the pupil. Methodological contributions advance some of the current proposals for inclusive education: importance of early intervention, value of interdisciplinary work, social integration of the individual as the ultimate goal of education. Despite the failure in the attempt to provide speech Victor, his proposals and claims for the right of a wild child to education served by administrators and scientists and sponsored by the government are a clear precedent of some basic proposals for inclusive education now guiding the main International Declarations.

Keywords: Itard; Inclusive education; Special education.

\section{Introducción}

La historia siempre ha sido maestra de la vida y desconocerla obliga a repetirla. Por eso una reflexión sobre figuras emblemáticas que han jalonado históricamente la educación de la humanidad aporta una clarificación acerca de los pasos de dicho proceso. Mucho más importante es el análisis que este estudio implica sobre el lugar que ocupamos en ese proceso y la necesaria revisión acerca de las circunstancias que lo condicionan, las indicaciones que deben orientarlo, así como de las metas últimas hacia las que guiarlo.

En este sentido, dirigimos nuestra reflexión hacia la obra de Jean Itard desde la perspectiva pedagógica. Concretamente desde las culturas, políticas yprácticas de la educación inclusiva, en la convicción de que esta etapa es el jalón último que ocupa la trayectoria que Jean Itard iniciara en el siglo XVIII junto a otros abanderados de la Educación Especial.

Su compromiso con la educación de un joven salvaje, en un momento en el que la educación universal empezaba a plantearse como un derecho en la Francia ilustrada,interpela los planteamientos que las Declaraciones Internacionales están formulando tres siglos después. Su sistematización del proceso de educación y rehabilitación orientan los planteamientos metodológicos, organizativos y didácticos con que aplicamos la educación inclusiva en nuestras aulas. Su implicación personal como educador cuestiona las actitudes y los valores con que los profesionales actuales, las familias y la sociedad en general afrontan el derecho a la educación completa y de calidad para todo el alumnado y, de forma especial, para aquellos cuya diversidad y necesidades rompen las monotonías de nuestros prejuicios y rutinas pedagógicas. 


\section{En clave de educación inclusiva}

Históricamente Jean Itard se enclava en lo que los historiadores de la educación especial clasifican como "la Era de las Instituciones". Durante toda la historia de la humanidad las personas con alguna alteración habían sido marginadas, excluidas, cuando no exterminadas (BERRUEZO; CONEJERO, 2009; SCHHEREMBERGER, 1984). Las limitaciones de la ciencia hasta la edad moderna, junto con los condicionantes religiosos, dificultaban las explicaciones científicas acerca de sus comportamientos. Son las propuestas ilustradas de la Revolución Francesa las que empiezan a demandar una educación universal. En el marco de esta ideología, con las aportaciones de la ciencia del siglo XVIII y las filosofías de Bacon, Descartes, Locke y Rousseau, es cuando aparecen las primeras instituciones educativas para la atención de personas con discapacidad, principalmente sordos (ABATE L'EPEE, 1760) y ciegos (HAÜY, 1784), que se extenderían por toda Europa a lo largo del siglo XIX.

La experiencia de Itard comparte las características de estos primeros intentos: iniciativa privada, ligada a instituciones filantrópicas de carácter asistencial y basada en avances significativos, principalmente desde el ámbito de la medicina. Su aportación, entre el magisterio de Pinel y su influencia en Seguín, le han granjeado el título de "padre de la Educación Especial" por su contribución a la educación de Víctor de L'Aveyron.

Un "largo camino" (BERRUEZO; CONEJERO, 2009) ha confirmado aquella inicial reivindicación de la educación universal ilustrada, hasta los planteamientos actuales, con la denominación de educación inclusiva. Entendemos por tal el cumplimiento efectivo del derecho a una educación completa y de calidad para todo el alumnado. Tal derecho inalienable debe atender de manera sustantiva las diversas necesidades personales - físicas, intelectuales, conductuales - y del contexto - familiares, sociales, económicas, ideológicas, étnicas, culturales. De modo que, debe aportar los recursos personales, organizativos, metodológicos y materiales precisos para atender de forma individualizada dichas necesidades educativas especiales. Por eso el aprendizaje dialógico, cooperativo y solidario (Freire, Freinet, Milani,...) constituyen elementos básicos de dicho proceso formativo. (AINSCOW, BOOTH; DYSON, 2006; ARNAIZ, 2003; CASANOVA; RODRIGUEZ, 2009; EADSNE, 2011; ECHEITA; AINSCOW, 2011; OPERTTI; BRADY, 2011; VEGA, LÓPEZTORRIJO; GARÍN, 2013).

La educación inclusiva se sustenta en los principios de igualdad de oportunidades, no discriminación, equidad, atención a la diversidad y accesibilidad universal. Precisa en sus fundamentos del establecimiento de una cultura de inclusión que incorpore a los sistemas educativos aquellas actitudes y valores propios de la atención a la diversidad: respeto y desarrollo de los derechos fundamentales,tolerancia, cultivo de las diferencias y solidaridad. (AINSCOW, 2007; BOOTH; AISCOW, 2000; ECHEITA, 2008; EADSNE, 2013; LÓPEZ-TORRIJO, 2009; LOPEZ-TORRIJO; CARNONELL, 2005).

Todas estas propuestas han sido sancionadas por sucesivas Declaraciones Internacionales de la UNESCO (DAKAR, 2000; JOMTIEM, 1990; MASCATE, 
2014 SALAMANCA 1996; ver en LÓPEZ-TORRIJO, 2009). Más recientemente la ONU proclamaba la Convención de los Derechos de las Personas con discapacidad,basada en los siguientes principios:

1 - El respeto de la dignidad inherente, la autonomía individual, incluida la libertad de tomar las propias decisiones, y la independencia de las personas;

2 - La no discriminación;

3 - La participación e inclusión plenas y efectivas en la sociedad;

4 - El respeto por la diferencia y la aceptación de las personas con discapacidad como parte de la diversidad y la condición humanas;

5 - La igualdad de oportunidades;

6 - La accesibilidad;

7 - La igualdad entre el hombre y la mujer;

8 - El respeto a la evolución de las facultades de los niños y las niñas con discapacidad y de su derecho a preservar su identidad.

El derecho a la educación de estas personas es recogido en el art. 25. En él se platea la educación inclusiva como elemento inherente al propio derecho a una educación, en los términos siguientes:

1 - Estados Partes asegurarán un sistema de educación inclusivo a todos los niveles así como la enseńanza a lo largo de la vida, con miras a:

2 - Desarrollar plenamente el potencial humano y el sentido de la dignidad y la autoestima y reforzar el respeto por los derechos humanos, las libertades fundamentales y la diversidad humana;

3 - Desarrollar al máximo la personalidad, los talentos y la creatividad de las personas con discapacidad, así como sus aptitudes mentales y físicas;

Hacer posible que las personas con discapacidad participen de manera efectiva en una sociedad libre.

En consecuencia los estados firmantes se comprometen a que todos los alumnos "puedan acceder a una educación primaria y secundaria inclusiva, de calidad y gratuita, en igualdad de condiciones con los demás, en la comunidad en que vivan [...] con el objetivo de la plena inclusión". Este derecho se hará extensivo "a la educación superior, la formación profesional, la educación para adultos y el aprendizaje durante toda la vida sin discriminación y en igualdad de condiciones con las demás".

De manera más inmediata la UNESCO ha dedicado la Declaración de su última reunión (Incheon, 2015) a plantear los objetivos de la educación para el 2030 
con un lema inequívoco: "Hacia una educacióninclusivay equitativa de calidad y unaprendizaje a lo largo de la vida para todos". En ella propone como objetivo general: "Garantizar una educación inclusiva, equitativa y de calidad y promover oportunidades de aprendizaje durante toda la vida para todos".

La Declaración se basa en una "concepción humanista de la educación y del desarrollo basada en los derechos humanos y la dignidad, la justicia social, la inclusión, la protección, la diversidad cultural, lingüística y étnica, y la responsabilidad y la rendición de cuentas compartidas." Por ello la educación, en cuanto derecho humano fundamental, es la base para la realización de otros derechos. A la vez, es esencial para la paz, la tolerancia, la realización humana y el desarrollo sostenible; para lograr el pleno empleo y la erradicación de la pobreza.

Destaca que una educación inclusiva y equitativa es la piedra angular para una "educación transformadora", que erradique "la exclusión y la marginación, las disparidades y las desigualdades en el acceso, la participación y los resultados de aprendizaje”.

Y subraya: "Ninguna meta educativa debería considerarse lograda a menos que se haya logrado para todos", especialmente para los más desfavorecidos: discriminados por motivos de género, discapacidad, población no escolarizada, en zonas de conflictos, violencia, desastres naturales...

La culminación de la educación inclusiva es una sociedad equitativa e inclusiva, en la que los valores y actitudes propios de la inclusión enriquecen la convivencia y desarrollo sociales (BARDY, HAYASHI, SCHLÜZEN, SEABRA, 2013; BOOTHS, 2000; CARBONELL, 2009; CARBONELL, LÓPEZ-TORRIJO, 2005; ECHEITA, 2006; EADSNE, 2011; OPERTTI, BRADY, 2011; UNESCO, 2008, 2015, VEGA, LÓPEZ-TORRIJO, 2011).

\section{Aportaciones de Jean Itard a la futura educación inclusiva}

Es preciso contextualizar la labor de Itard para ponderar el alcance de sus propuestas. Si bien cronológicamente Itard es uno de los jalones iniciales de la Educación Especial y un pionero de la denominada "Era de las Instituciones", el contraste con las propuestas de su contexto lo sitúa, en varios aspectos, como un precursor de la educación inclusiva. Comentamos algunos significativos:

\section{Se fundamenta en una filosofía equitativa de la educación:}

En un contexto en el que la mayoría de la población infantil no tenía acceso real a la educación, los niños y niñas en condiciones desfavorecedoras (mujeres, personas discapacitadas, pobres, minorías étnicas,...) apenas disponían de experiencias formativas. El caso concreto de Victor de Aveyron cuenta con más ciudadanos que lo observan como un espectáculo de feria, que aquellos que plantean para él un trato normalizado. Incluso un primer diagnóstico médico, realizado por Pinel, lo clasifica como "idiota" y, en consecuencia, ineducable.

Sólo una mente adelantada a su contexto, con una concepción profunda de los derechos de toda persona,podía reivindicar que un "salvaje" "nao é como geral- 
mente se acredita, um imbécil semesperanças, mas um ser interessante, que merece, sob todos os aspectos, a atençao dos observadores e os cuidados especiais que lhederamumaadministraçao pública esclarecida e filantrópica”(ITARD, 1801, p.127). Esta preocupación por los más excluidos, este reconocimiento del derecho a una educación para ellos, esta demanda de que el estado asuma su responsabilidad equitativa en el plano educativo, fundamenta los principios de una educación inclusiva de forma especialmente valiosa e innovadora en un contexto tan opuesto como el del siglo XVIII, y adelanta las reivindicaciones que la UNESCO sigue repitiendo tres siglos después.

Por eso Itard puede concluir después de cuatro años de duro trabajo que, más allá de su radical gusto por la libertad y la vida en el medio natural, Víctor ha alcanzado un nivel de socialización inimaginable para una persona aislada de la sociedad: "se mostra reconhecido pelos cuidados que se têm com ele, suscetível de uma amizadecarinhosa, sensível ao prazer de agir certo, envergonhado de seus enganos e arrependido de suas impetuosidades" (ITARD, 1807, p. 229).

Por eso mismo concluye su informe de 1807 reclamando para esos seres

desertados pela natureza, rejeitados pela sociedade e abandonados pela medicina, os cuidados que lhe foram dados, aqueles que lhe devem ainda, as mudanças que ocorreram, aquelas que se podem esperar, a voz da humanidade, o interesse inspirado por um abandono tấo absoluto e um destino tão estranho, tudo recomenda esse rapaz extraordinário à atenção dos cientistas, à solicitude de nossos administradores e à proteção do Governo. (ITARD, 1807, p. 229).

\section{Realiza un diagnóstico globalizado, pedagógico y funcional}

Itard parte del anterior dictamen médico que consideraba que "não era suscetível de espécie alguma de sociabilidade e de instrução" (p. 132).

No contaba la pedagogía a finales del siglo XVIII con las pruebas psicométricas actuales. Pero, fruto de su observación, Itard identifica en Víctor sus limitaciones sensitivas (tacto, olfato, y especialmente oído) y de memoria; su aptitud para imitar; la inestabilidad de su humor; su insensibilidad al afecto; su dependencia absoluta de la satisfacción de sus necesidades animales. En consecuencia, lo califica como un desarrollo equivalente a un niño de diez o doce meses de edad por sus hábitos antisociales, la completa desatención, la escasa flexibilidad de algunos de sus órganos, una sensibilidad "accidentalmente embotada", los movimientos convulsos y ruidosos; y por su escasa sensibilidad al frio y calor, junto a una finísima sensibilidad para localizar los alimentos que precisa.

Por ello, concluye que estas características y su grado de inteligencia serían las de cualquier otro adolescente que hubiera sido privado desde la infancia de toda educación y que hubiese vivido aislado de los individuos de su especie. Es, pues, el contexto, al igual que señalan actualmente algunos colectivos de personas con discapacidad, el que condiciona su desarrollo. Por tal debe ser el eje de la intervención educativa que se programa para él. 


\section{Diseña un plan sistemático, global e inclusivode intervención} partiendo de las necesidades educativas especiales del alumno,en un contexto de normalización

En todo momento Itard parte de las necesidades educativas del joven, que considera fruto de su aislamiento "antinatural". Alude reiteradamente a señalar en qué medida sus limitaciones comunicativa, intelectual y afectiva, su desadaptación social, así como su carácter "egoísta" son fruto de su infancia salvaje.

Es en base a dichas limitaciones como diseña un plan minucioso para desarrollar sus capacidades de forma global y según los ámbitos propuestos por la pedagogía de su tiempo (Locke): ámbitos físico, intelectual y moral. Y lo sistematiza en cinco metas normalizadoras:

"Primeira meta: interessá-lo pela vida social, tornando-a mais amena do que aquela que ele entáo levava, e sobretudo mais análoga à vida que acabava de deixar.

Segunda meta: despertar a sensibilidade nervosa com os mais enérgicos estimulantes e algumas vezes com as vivas afiçoes da alma.

Terceira meta: ampliar a esfera de suas ideias dando-lhe necessidades novas e multiplicando suas relaçôes com os seres que o circundavam.

Quarta meta: levá-lo ao uso da fala, determinando o exercício da imitaçao pela lei imperiosa da necessidade.

Quinta meta: exercitar durante algum tempo, a partir dos objetos de suas necessidades físicas, as mais simples operaçôes da mente e determinar depois sua aplicação aos objetos de instruçáo".

Con igual intuición Itard elige para Víctor un contexto de educación normalizado, pese a que el propio educador era médico en el Instituto Nacional de Sordo -mudos que fundara en 1760 el Abate L'Epee (1776). Así, en lugar de internarlo en el Instituto, encarga su cuidado personal a Mme. Guerin, ocupándose él mismo de su educación.

\section{Adapta la metodología y recursos a las capacidades, limitaciones y necesidades de Víctor}

Itard es consciente de la importancia de la atención temprana cuando afirma: "esquecendo que nossos órgãos ficam ainda menos flexíveis e a imitação ainda mais difícil quando o homem está afastado da sociedade e da época de sua primera idade (ITARD, 1801, p. 130). De hecho, la mayor dificultad para el aprendizaje que identifica en Víctor consiste en no haber contado con dicha estimulación durante su primera infancia.

Piensa que en los años iniciales de la infancia, al igual que en los animales, la propia subsistencia lleva al niño a intentar cubrirsus necesidades primarias. Sin embargo, cuando avanza en su madurez, aparecen las capacidades que los distinguen de los restantes animales: "sua atenção, sua memoria, seu juízo" (ITARD, 1801, p. 165). Víctor, carente de la estimulación inicial, no sentía otro interés que el de seguir 
cubriendo sus necesidades primarias. Por ello, Itard aborda un proceso de aprendizaje que compense las carencias de su infancia.

Respecto a la estimulación física, y siguiendo a Locke y Condillac, la refirió fundamentalmente a los sentidos, como fuentes en la formación y desarrollo de las ideas. La inició por el sentido del oído, al entender que él concurre de forma particular en la formación de las ideas. Por su especificidad, abordaremos este tema en el apartado 3. Sí adelantamos que el fracaso que recogió con dicha estimulación le llevó a pasar al sentido de la vista.

Tras un duro proceso y refiriendo su estimulación a las palabras, consiguió la discriminación completa y rápida de las formas escritas de aquellas, pero "sem as pronunciar e mesmo sem lhes conhecer a signifição" (p. 192), algo que Itard nunca llegó a entender.

En cuanto al tacto, planificó el entrenamiento en la similitud y diferencia del calor, formas, etc., especialmente rápida en la identificación de los alimentos. Una vez adquirida, la aplicó a la diferenciación de las letras, que presentaba en dibujos, cartulinas y formas metálicas.

Respecto al gusto, desarrolló la diferenciación de los sabores, comidas, licores completamente nuevos para Víctor. En todo momento éste mostró su preferencia por el agua, el sol y los alimentos naturales con los que se había criado.

Pero el desarrollo de los sentidos para Itard sólo estaba en función del desarrollo intelectual. Desde esta concepción, planificó la comprensión de los conceptos de objetos concretos mediante la asociación de dicho objeto real a su dibujo. Posteriormente, la asociación la extendía a la grafía de la palabra, pero Victor resolvía dichas asociaciones mediante la memoria y no por la comprensión. Entonces Itard intentó que diferenciara los conceptos de formas y colores mediante la combinación de figuras geométricas policromadas. A continuación, fue sustituyendo las figuras completas por las letras, que Víctor aprendía como figuras en relieve memorizando sus formas. En un paso siguiente llegó a diferenciar la totalidad de las letras que componían diversas palabras y a asociarlas globalmente a los objetos, pero no sólo mediante la memoria visual. De hecho sólo asociaba el nombre al objeto de la muestra, pero no a otro de la misma especie. Víctor no conseguía la generalización del concepto, sino la pura asimilación visual. Intentó entonces enseñar las características comunes de los objetos para facilitar la generalización del concepto. Una misma palabra escrita la refería a dos objetos que cumplían la misma función.

Procuró estimular el poder dela inventiva, para desarrollar la comprensión del significado de las palabras. Utilizando las letras metálicas y su ordenación en palabras, le enseñó a diferenciar las partes de un todo (adjetivos), con sus respectivos nombres; las características de tamaño de los distintos objetos; las acciones que implicaban las palabras (verbos). Intentó adiestrarlo en la escritura, pero, falto de su comprensión, debió recurrir a la pura imitación de los gestos necesarios para su composición. Con ello, Víctor repetía los gestos de Itard y "escribía". Pasados unos meses, Víctor copiaba de seguido palabras, cuyo trazado había memorizado; luego las reproducía de memoria en su 
forma escrita y las utilizaba para "exprimir suas necessidades, solicitar os meios de satisfaze-las e aprender pela mesma via a expressão das necessidades ou da vontade dos outros" (ITARD, 1807, p. 213). Ensayó nuevas técnicas y materiales para favorecer la asociación y con ella la comprensión de los conceptos escritos, pero Itard concluye que su práctica es resuelta por puros ejercicios de imitación. En todos estos pasos se vislumbra una estrategia coherente del aprendizaje, ceñida a las limitaciones de Víctor y basada en las teorías e intuiciones del aprendizaje propias de su momento, pero llevadas a cabo de forma lógica y sistemática.

Combinaba, además, la estimulación de la emulación y los premios con la disciplina y el castigo, pero siempre argumentando el uso de cada uno de ellos en un determinado contexto.

En suma, Itard programó y llevó a cabo una estimulación y desarrollo de todos los sentidos, diferenciando sus sensaciones y su escritura, pero sin alcanzar hacerle entender el valor representativo de los signos escritos (decodificación), ni su significado (semántica).

Con todo ello, Itard concluye que "o selvagem de L'Aveyron, é dotado do livre exercício de todos os seus sentidos; que da provas contínuas de atençáo, de reminiscência, de memória; que pode comparar, discernir e julgar, aplicar enfim todas as faculdades de seu entendimento a objetos relativos à sua instrução" (ITARD, 1801, p. 174).

Finalmente y en cuanto al desarrollo de las facultades afectivas, Víctor manifestaba inicialmente los sentimientos propios de un recién nacido, referidos únicamente al instinto de supervivencia. Insensible, inactivo, sucio y ajeno a los afectos de su propia cuidadora. Mediante un diseño pormenorizado de experiencias prácticas Itard consiguió hacer que aparecieran en él los sentimientos de satisfacción al conseguir los aprendizajes o al recibir elogios; impotencia cuando ello no le era posible; alegría al reencontrarse con las personas que le cuidaban; justicia ante su práctica de sustraer comida; injusticia ante tratos inadecuados frente a su buena conducta. Sin embargo, en todo momento prevalece en él el sentimiento de egoísmo y es ajeno a la piedad y al valor del "sacrificio" que hacenpor él las personas encargadas de su cuidado.

Sorprende a Itard la nula reacción de Víctor ante su contacto con el sexo distinto y el escaso efecto que produce en él la aparición de la pubertad.

\section{Evaluación sistemática de los resultados del aprendizaje}

El rigor metodológico de Itard - ajustado a las limitaciones de su momentoculmina con la sistematización del seguimiento y la objetividad en la valoración de los resultados que concluye: "Impus-me o dever de apresentá-los sem distinçáo, tanto uns como os outros, e relatar com a mesma veracidade meus revezes bem como meus sucessos" (ITARD, 1807, p. 228)

En un momento histórico en el que era tan frecuente sobredimensionar los logros de los "adelantos" con los que algunos investigadores se presentaban ante la Academia Real de las Ciencias, Itard hace un relato completamente imparcial de sus 
resultados. Con la misma objetividad habla de los logros conseguidos en los aprendizajes de las letras, o de los esfuerzos realizados por Víctor, como de la falta de socialización, desarrollo afectivo o adquisición del habla.

Muestra ejemplar de ello esel balance que presenta de su alumno cuando concluye que: 1 - la educación de este joven es incompleta a consecuencia de la "nulidadequase absoluta dos órgâos da audiçâo e da fala"; 2 - las facultades intelectuales se desarrollan de forma "lenta e penosa" por culpa de la inacción y los resultados exigen grandes esfuerzos; 3 - que prevalece el egoísmo frente a otros sentimientos y que la relación entre las necesidades de los sentidos y los afectos del corazón son fruto de una educación que Víctor no ha podido alcanzar.

Pese a todo, concluye su informe con una propuesta positiva: 1 - el perfeccionamiento de la vista, del tacto y del gusto contribuirían poderosamente al desarrollo de sus facultades intelectuales; 2 - considerando su evolución general, se valora positivamente:

o conhecimento do valor convencional dos signos do pensamento, a aplicação desse conhecimento à designação dos objetos e a enunciaçấo de suas qualidades e de suas açóes, donde a extençáo das relaçóes do aluno com as pessoas que o cercam, a faculdade de lhes expressar suas necessidades, de receber ordens delas e de fazer com elas uma livre e contínua troca de pensamentos. (ITARD, 1807, p. 229).

3 - más allá de su preferencia por la vida en libertad en el medio natural, Víctor ha empezado a manifestar agradecimiento por los cuidados recibidos, sensibilidad hacia la amistad cariñosa y sensible al placer de acertar, a avergonzarse de sus errores $\mathrm{y}$ arrepentimiento por sus impulsos.

Consciente de las limitaciones y potenciales de la ciencia concluye con una propuesta de intervención interdisciplinar e individualizada:

a marcha do ensino pode e deve-se esclarecer com as luzes da medicina moderna, que, de todas as ciências naturais pode cooperar com mais força para o aperfeiçoamento da espécie humana, apreciando as anomalias orgânicas e intelectuais de cada indivíduo e determinando assim o que a educação pode fazer por ele e o que a sociedade pode esperar dele. (ITARD, 1801, p. 176).

\section{El problema del habla}

El habla de Víctor fue a la vez el principal objetivo de Itard y su mayor frustración. Consciente de que el desarrollo intelectual se producía por medio del habla, centró en ella su mayor esfuerzo. Sin embargo, tenía ante sí dos graves dificultades. La primera, la falta de estimulación durante la infancia. No podía conocer Itard la existencia del "periodo crítico" para la adquisición de la fonología, comprendido entre los 0 y 3 años, pero lo intuía al afirmar que "nossos órgãos ficam ainda menos flexíveis e a imitação ainda mais difícil quando o homem está afastado da sociedade e da época de sua primeira idade" (ITARD, 1801, p. 130). 
La segunda dificultad era la falta de audición. Itard confunde la nula comprensión del habla con la falta de audición; y cree que la ausencia de la fonación en la infancia de Víctor ha inutilizado sus órganos, por lo que éste no consigue sobrepasar la emisión de los sonidos guturales, al igual que los alumnos del Instituto Nacional fundado por L'Epee, del que era médico Itard.

El más pormenorizado esfuerzo de Itard se centró en estimular la audición y comprensión del habla en Victor. Sorprende que no utilizara el método de L'Epee, quien partía de la dactilología para acabar desarrollando la lenguade signos. Sería necesario analizar con más extensión la minuciosagraduación del proceso, la combinación de materiales y estímulos: entrenamiento auditivo con los ojos cerrados; discriminación de sonidos de objetos similares y de voces humanas; intento de comprensión del significado de las palabras escritas, asociándolas a los objetos que representan; copia de escritura; asociación de las palabras escritas a las características y acciones de los objetos representados; nada le permitió enseñar el valor representativo de las palabras y su significado.

Es comprensible que Itard no conociera los resultados conseguidos por la metodología oralista (LOPEZ-TORRIJO, M. 2005), originada en Espańa enel siglo XVI por Fray Pedro Ponce de León y publicada por Juan Pablo Bonet (1620). Pero resulta difícil entender cómo no llegó a conocer los resultados, igualmente oralistas, expuestos por el judío español Jacobo Rodríguez Pereira en 1746 en la Academia de Caen y más tarde ante la Academia Real de Ciencias en París (SEGUIN, 1932), que le granjearon tan enorme fama y fortuna.

Itard era consciente de que el aprendizaje definitivo del hombre es el habla. Sabía que había pasado el tiempo de su mejor aprendizaje: la primera infancia. Estaba erróneamente convencido de que Víctor no podía adquirir el habla por la vía auditiva. La estimulación visual que probó no le permitió alcanzar más que una simple imitación de las posiciones y movimientos de todos los órganos de la fonación visibles. Tras un año de imitación. Víctor no conseguía emitir más que algunos monosílabos confusos.

Fue, en definitiva, la falta del desarrollo de la fonología, imprescindible para alcanzar una comprensión lectora (decodificación fonológica),lo que hizo concluir a Itard: "resignei-me a terminar ali minhas derradeiras tentativas em prol da fala e abandonei meu aluno a um mutismo incurável” (ITARD, 1807, p. 216).

\section{Conclusiones}

Los Informes presentados por Itard en 1801 y 1807 acerca de su trabajo con el joven salvaje de L'Aveyron le han granjeado el reconocimiento universal como "padre de la Educación Especial"

Una interpretación de los mismos desde la perspectiva de la educación Inclusiva nos permiten considerarlo igualmente como uno de los más lejanos predecesores de ésta por cuanto: 
- Reivindica el derecho a la educación de los más desfavorecidos.

- Lleva a cabo un programa de educación integral (física, intelectual y moral) acorde con los conocimientos de su tiempo.

- Desarrolla un proceso de rehabilitación individualizado, partiendo de un diagnóstico global y atendiendo a las capacidades, limitaciones, necesidades e inquietudes de su alumno.

- Realiza, desde su contexto histórico, valiosas aportaciones teóricas necesarias para una educación inclusiva de calidad: importancia de la atención temprana; valor de una trabajo interdisciplinar; integración social de los individuos, como última meta de la educación.

- Concluye, pese a las limitaciones de su experiencia, la necesidad de la atención de los administradores, científicos y el Gobierno al servicio del desarrollo integral de los más marginados.

\section{Referências}

AINSCOW, M. Taking an inclusive turn. Journal of Research in Special Educational Needs. v. 7, n. 1, p. 3-7, 2007.

AINSCOW, M.; BOOTH, T.; DYSON, A. Improving Schools, Developing Inclusion. New York: Routledge, 2006.

ARNAIZ, P. La educación inclusiva: una escuela para todos. Málaga: Ediciones Aljibe, 2003.

BERRUEZO ALBÉNIZ, M. R.; CONEJERO LÓPEZ, S. El largo camino hacia la educación inclusiva: la educación especial y social del siglo XIX a nuestros días. XV Coloquio de Historia de la Educación, Pamplona-Irueña. Pamplona: Universidad de Navarra, 2009.

CASANOVA, M. A.; RODRÍGUEZ, H. Educación inclusiva, un horizonte de posibilidades. Madrid: Edit. La Muralla, 2009.

ECHEITA, G. Inclusión y exclusión educativa: “voz y quebranto”. REICE. Revista electrónica Iberoamericana sobre calidad, eficacia y cambio en Educación, v. 6, n. 2, p. 9-18, 2008.

ECHEITA, G.; AINSCOW, M. La educación inclusiva como derecho. Marco de referencias y pautas de acción para el desarrollo de una revolución pendiente. Tejuelo, n. 12, p. 26-46, 2011.

EADSNE. Mapping the implementation of policy for inclusive education - an exploration of challenges and opportunities for developing indicators a European Agency for Development in Special Needs Education. Bruselas, EADSNE, 2011. Disponível em: <http://www.european-agency.org/publications/flyers/mipie/MIPIE-summary-of-proposalsEN.pdf/view>. Acesso em 26 ago 2016.

ITARD, J. De l'education d'un homme sauvage ou des premiers developpmens hysiques et moraux du jeune sauvage de l'Aveyron. Paris: Goujon fils Imprimeur - Libraire, 1801. In: BANKS-LEITA, L.; GALVAO. I. A educaçáo de um selvagem. As experiências pedagógicas de JEAN ITARD. Sâo Paulo: Cortez Editora, 2000.

Raport fait a son Excellence le Ministre de l'Interieur sur les nouveaux developpemens et l'etat actuel du sauvage de l'Aveyron. Paris: Imprimiére Imperaile, 1807. In: BANKS-LEITA, L.; GALVAO. I. A educaçáo de um selvagem. As experiências pedagógicas de JEAN ITARD. São Paulo: Cortez Editora, 2000.

L'EPÉE, C. M. Institution des sourds et muets par la voie des signes methodiques. Paris: Librairie Nyon, 1776.

LÓPEZ-TORRIJO, M. El derecho a una educación (inclusiva) de las personas con discapacidad en las Declaraciones Internacionales. In: BERRUEZO ALBÉNIZ, M. R.;CONEJERO LÓPEZ, S. El largo camino hacia la educación inclusiva: la educación especial y social del siglo XIX a nuestros días. XV Coloquio de Historia de la Educación. Pamplona: Universidad de Navarra, v. 1, 2009, pp.161-170. 
LÓPEZ-TORRIJO, M. La educación de las personas con sordera. La escuela oralista espańola. Valencia: Universitat de València, 2005.

LOPEZ-TORRIJO, M.; CARBONELL, R. (Coords.). La integración educativa y social. Barcelona: Ariel, 2005.

OPERTTI, R.; BRADY, J. Developing inclusive teachers from an inclusive curricular perspective. PROSPECTS, v. 41, n. 3, p. 459-72, 2011.

PABLO BONET, J. Reduction de las letras y arte para enseńar a ablar los mudos. Madrid: Imp. de francisco Abarca de Ángulo, 1620.

SCHHEREMBERGER, P. H. D. Historia del retraso mental. San Sebastián: SIIS,1984.

SEGUIN, E. Jacobo Rodríguez Pereira. Primer maestro de sordomudos en Francia. Biografía y análisis de su método. Madrid: Francisco Beltrán, 1932.

. Declaración Mundial sobre Educación para todos. Jomtien, Tailandia. 1990. Disponível em: <http:// www.unesco.org/education/pdf/JOMTIE_S.PDF>. Acesso em 26 ago 2016.

Foro Mundial sobre la Educación. Informe final. Dakar, Senagal. 1990. Disponível em: <http:// unesdoc.unesco.org/images/0012/001211/121117s.pdf >. Acesso em 26 ago 2016.

Declaración de Salamanca y Marco de acción sobre Necesidades Educativas Especiales. Conferencia Mundial de la UNESCO sobre necesidades educativas especiales: acceso y calidad. Salamanca, 7 al 10 de junio de 1994. Disponível em: <http://www.unesco.org/education/pdf/SALAMA_S.PDF>. Acesso em 26 ago 2016.

El acuerdo de Mascate. Declaración final de la Reunión Mundial sobre la EPT de 2014. Reunión Mundial sobre la Educación para Todos. Mascate (Omán), 12-14 de mayo a 2014. Disponível em: <http:// www.unesco.org/new/fileadmin/MULTIMEDIA/FIELD/Santiago/pdf/Muscat-Agreement-ESP.pdf>. Acesso em 26 ago 2016.

Foro Mundial de Educación, Incheon (República de Corea). 2015. Disponible en: <http://www. unesco.org/new/es/education/themes/leading-the-international-agenda/education-for-all/world-education-forum-2015/regional-conferences/>. Acesso em: Acesso em 26 ago 2016.

VEGA, A., LÓPEZ-TORRIJO, M.; GARÍN, S. Educación inclusiva: entre la crisis y la indignación. Intersticios, v. 7, n. 1, p. 315-336, 2013.

\section{Correspondência}

Manuel Lopez-Torrijo - Fac. de Filosofía y Ciencias de la Educación. Av. Blasco Ibáńez, 30. 46010, Valencia, Espanha.

E-mail: lopezm@uv.es - garfran2@alumni.uv.es - jelogar@alumni.uv.es

Recebido em 03 de junho de 2016

Aprovado em 06 de agosto de 2016 